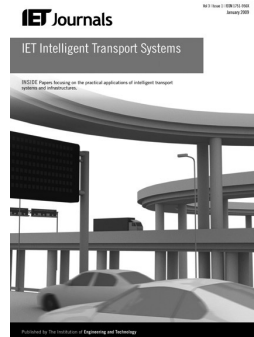

\title{
GuideWeb: a conceptually infrastructure-free vehicle navigation system
}

\author{
B.X. Weis A. Sandweg \\ BlackForestLightning, Haeberlinstr 29b, 70563 Stuttgart, Germany \\ E-mail: bernd.weis@blackforestlightning.de
}

\begin{abstract}
A new paradigm named GuideWeb for networks based on vehicle-to-vehicle (v2v) communication is presented. GuideWeb is a support network for vehicle navigation and guidance. It is constituted by the cooperation of a multitude of autonomous MapSynthesiser nodes located in vehicles. A MapSynthesiser, being the autonomous core of GuideWeb, receives over radio communication traffic flow information using information-enhanced maps (called map syntheses) from other GuideWeb participants' MapSynthesiser. From received map syntheses and the information of its own travel route it creates a new map synthesis, which is then broadcasted. MapSynthesiser provides timely and accurate information on traffic flow and density as well as traversability everywhere within a radius of $\sim 100 \mathrm{~km}$ to a navigation system for driver assistance. MapSynthesiser cooperation is based on short-range radio communication (e.g. Wireless Local Area Network - WLAN) and/ or mobile communication networks. GuideWeb deploys the simple method of broadcast for network formation and thus, avoids the time lags arising from setting up and maintaining a connection-oriented ad hoc network. By the map-based data design, data security and privacy are ensured. Therefore, GuideWeb can serve as a commercially viable introductory phase for full-fledged $\mathrm{v} 2 \mathrm{v}$ communication networks. The lessons learned from GuideWeb deployment provide insights for a v2 $\mathrm{v}$ network design.
\end{abstract}

\section{Introduction and background}

There is an increasing demand for individual traffic and route information and navigation indicated by the still-rising shipments of navigation devices and/or navigation software on standard platforms like smartphones with the related services for real-time traffic information. The existing systems are typically based either on elaborate infrastructures for traffic measurement or on traffic patterns derived, for example from individual mobile phone movements. Both concepts have their drawbacks.

The first concept requires substantial investment into setting up the infrastructure, its maintenance incurs cost and in the case of a privately run enterprise should result in some profits. Therefore, this service for the end user cannot be free of charge. Furthermore, the measurement infrastructure is typically only available on motorways.

The second concept collects data by tracking individual user-movements, for example mobile phones. Processing this data and matching it to maps allows the derivation of traffic flow estimations. This process cannot ensure privacy as patterns of movement of individuals are recorded. The potential misuse of this data mandates a rigid supervision of the process by independent auditors. Again, the effort to put these concepts into place incurs capital expenditures in computing infrastructure and operational cost.

$\mathrm{v} 2 \mathrm{v}$ communication offers a remedy for these drawbacks. First, v2v communication allows, in addition to navigation, a variety of different other applications, for example communicating dangerous situations to other vehicles [1-3] and/or mobile Internet [4, 5] with their corresponding security problems [6] ands so on. There are different proposals for $\mathrm{v} 2 \mathrm{v}$ communication under standardisation, for example IEEE 802.11 , CALM set of ISO standards. A German study [7] indicates that v2v networking will not be in place before 2020, although the European Union supports a variety of projects with this target [8-12].

Very often, especially in Europe, the propensity of customers to spend money for services on a recurrent basis is rather low; they prefer a one-time payment if necessary. Finally, the quality of data sets the price customers are willing to pay for this service.

This sets the scene for the concept presented here. The requirements derived from above are:

- to create most up-to-date traffic flow information and navigation support everywhere (ubiquity);

- to offer most up-to-date information on temporarily traversable/non-traversable routes not provided by the maps of a navigation system;

- to ensure privacy and data security;

- to avoid recurrent service charges.

The implications of these requirements are manifold. The most important one is: No investments into infrastructure and cost for system maintenance. Making the reasonable assumption of there being no public subsidy, the direct 


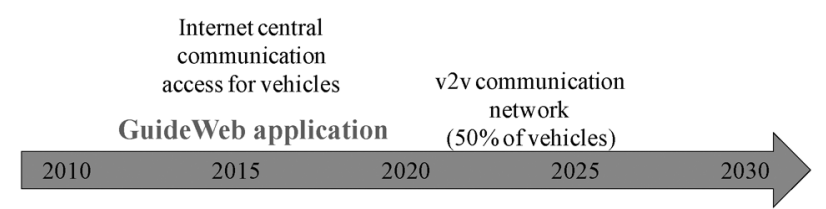

Fig. 1 Time line of $v 2 v$ communication introduction: GuideWeb positioning

structural implication is that no centralised processing, evaluation or distribution unit must be required for the functioning of the process.

The concept GuideWeb presented in the following fulfils these requirements. GuideWeb is a systemic process, in which a multitude of participants are involved. It facilitates the transmission and reception of information of vehicles using a radio transmitter and receiver and is based on a device, which processes the information received and enables the presentation of synthesised information to the user. A similar concept has been presented in [13]. Since communication is based on simple broadcast, GuideWeb communication technology is easy to implement, and can therefore serve as an intermediate introductory step in the full-fledged $v 2 \mathrm{v}$ networking with a complete communication protocol (see Fig. 1) that is technologically and commercially viable.

This study describes the functioning and implementation of GuideWeb. First the concept of GuideWeb and the autonomous MapSynthesiser as its constituting element is introduced, followed by a description of how GuideWeb/ MapSynthesiser fits into a driver-assistance environment. The next two sections present the MapSynthesiser and its map processing capabilities. Simulation results that quantify penetration requirements and a conclusion complete the study.

\section{Guideweb}

The principle of GuideWeb is intriguingly simple - one gets information, processes it, uses it and distributes the processed information. Information is freely offered and everyone can take it. GuideWeb is constituted by a multitude of participants where the participants continuously broadcast their information and knowledge about current traffic and environment in the form of map syntheses. A map synthesis is the data of the synthesised map in compressed form (NowMap) derived from the aggregation of all routes GuideWeb participants have traversed, including averaged speed and traffic density information of route segments. Thus, the individual map synthesis is built up from every participant's best knowledge of traffic flow and environment. In order to facilitate this process each participant is equipped with a MapSynthesiser of which the functional diagram is shown in Fig. 2.

The MapSynthesiser is a software application (executing together with a navigation application), which autonomously enables the functionalities of the GuideWeb only requiring connectivity from the communication platform and some basic vehicle information. MapSynthesiser provides timely and accurate information on traffic flow and density as well as traversability of roads everywhere within a radius of $\sim 100 \mathrm{~km}$. This information can be further processed and displayed to the driver, for example by a navigation system. The MapSynthesiser generates the NowMap, the representation of the current information and knowledge about the environment, from which the broadcasted map synthesis is derived.

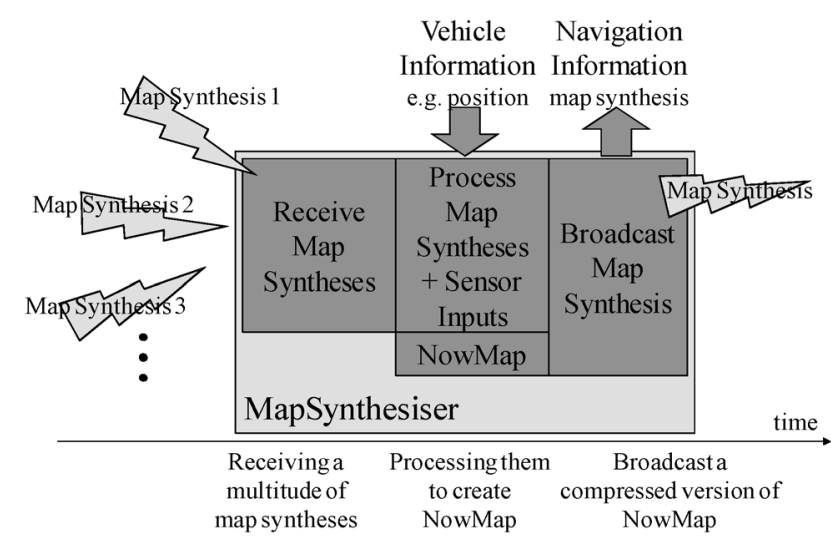

Fig. 2 Functional diagram of the MapSynthesiser

Conceptually GuideWeb is self-organising, self-contained and independent of any infrastructure. Functionally, GuideWeb rests entirely on the cooperation of the multitude of autonomous MapSynthesiser nodes [14], which constitutes the system's fault resilience. If a MapSynthesiser node fails it will cease to participate in GuideWeb which is sufficiently maintained by the other MapSynthesiser nodes. When the failed node has recovered it will continue participating. However, GuideWeb results may be used by city traffic authorities for traffic flow control.

\section{Environment for MapSynthesiser operation}

MapSynthesiser typically operates in a driver-assistance environment and Fig. 3 shows how MapSynthesiser fits into this. MapSynthesiser exploits the $\mathrm{v} 2 \mathrm{v}$ communication platform to send and receive messages. Two message types have been defined as below.

Synthesis messages contain a data-condensed version of NowMap, and additional information, for example road conditions, points of interest and so on when available and appropriate. On the incoming side synthesis messages comprise the data compressed NowMaps of other GuideWeb participants' MapSynthesisers, on the outgoing side its own NowMap. From the incoming synthesis messages the corresponding map data are extracted and made available for further processing.

Presence messages contain a minimum of information indicating the presence of a MapSynthesiser. Presence messages are very short and from their reception together with the reception of synthesis messages an estimate of traffic density can be derived. Furthermore, in phases of very low MapSynthesiser density, that is traffic density, presence messages serve as a trigger to broadcast synthesis messages; if no MapSynthesiser is in radio reach to receive synthesis messages they will not be transmitted.

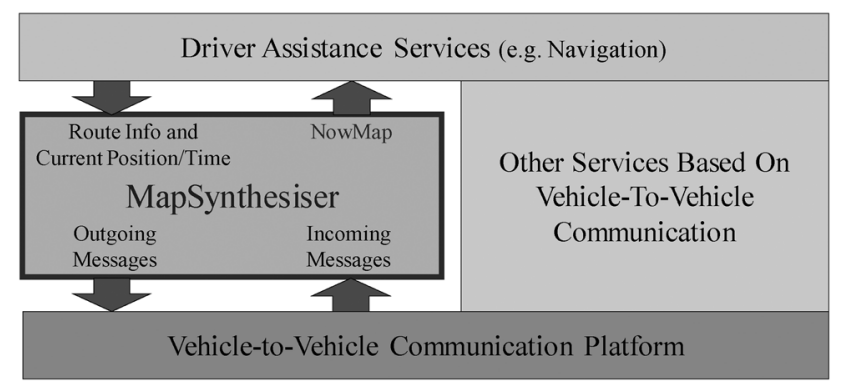

Fig. 3 MapSynthesiser in the driver-assistance environment 
Further, MapSynthesiser uses driver assistance services for positioning as well as time information and optionally for a map of the environment of the navigation system. This map may then serve as the basis for the map processing in the MapSynthesiser. The map representation in MapSynthesiser is based on Earth coordinates to ensure independence from the map representation of the navigation system [15]. The navigation system receives a map representation with attributes for route segments - these attributes being most prominently traffic flow, traffic flow trend estimation as well as traffic density. These attributes are time-stamped.

\section{Outline of MapSynthesiser}

The MapSynthesiser comprises the functionalities as described in the following based on the block diagram shown in Fig. 4. The MapSynthesiser broadcasts its messages in two modes:

- Broadcast Mode 1 it broadcasts a synthesis message;

- Broadcast Mode 2 it broadcasts a presence message.

In addition to the flow control mechanisms of the $\mathrm{v} 2 \mathrm{v}$ communication platform that are typically based on a carrier sense multiple access/collision avoidance (CDMA/CA) mechanism, stochastic processes control the broadcast modes to ensure a maximum throughput of messages. These stochastic processes employ the same mechanism: A synthesis (presence) message is sent on average every $T_{\mathrm{S}}\left(T_{\mathrm{P}}\right)$ seconds where the time to send is determined randomly from a uniform distribution with mean $T_{\mathrm{S}}\left(T_{\mathrm{P}}\right)$, respectively. If a synthesis message cannot be sent at that time, it is backlogged by a time, which again is randomly chosen with a smaller mean $T_{\mathrm{SB}}$. If there are an increasing number of backlogs indicating high transmission activity, $T_{\mathrm{S}}$ is accordingly increased, and vice versa. Typical values to start with are $T_{\mathrm{S}}=8 \mathrm{~s}, T_{\mathrm{P}}=1 \mathrm{~s}$ and $T_{\mathrm{SB}}=2 \mathrm{~s}$ corresponding to 160,20 and $40 \mathrm{~m}$ travelled at $20 \mathrm{~m} / \mathrm{s}$ speed, which allow for sufficient change in the receiving MapSynthesisers. This mechanism together with the platform flow control allows sufficient congestion control. The uniform distribution has been chosen to allow maximum spread within the given limits.

It is interesting to note that the system is 'self-scaling' because of the restricted bandwidth availability. When there are many GuideWeb participants in the vicinity, the probability increases that a MapSynthesiser is not able to send its information at its specified time. However, because of the system-inherent redundancy (recall the properties of NowMap) the abundant information available - even the information generated by the backlogged MapSynthesiser the previous transmissions - will more than enough compensate.

The v2v communication platforms communicate either

- directly by broadcast on some carrier frequency typically in the 2.4 or $5 \mathrm{GHz}$ band reserved for short-range applications (WLAN - Wireless Local Area Network) or

- by mobile networks' cell broadcast where the mobile network (global system for mobile communications (mobile communication standard) (GSM), universal mobile telecommunications system (UMTS), long term evolution (LTE) and so on) base stations receive broadcast messages from and distribute them to the vehicles within range or

- a combination thereof.

With respect to WLAN communication all MapSynthesiser radio subsystems transmit and receive information on the same carrier frequency on the same channel and employ the same

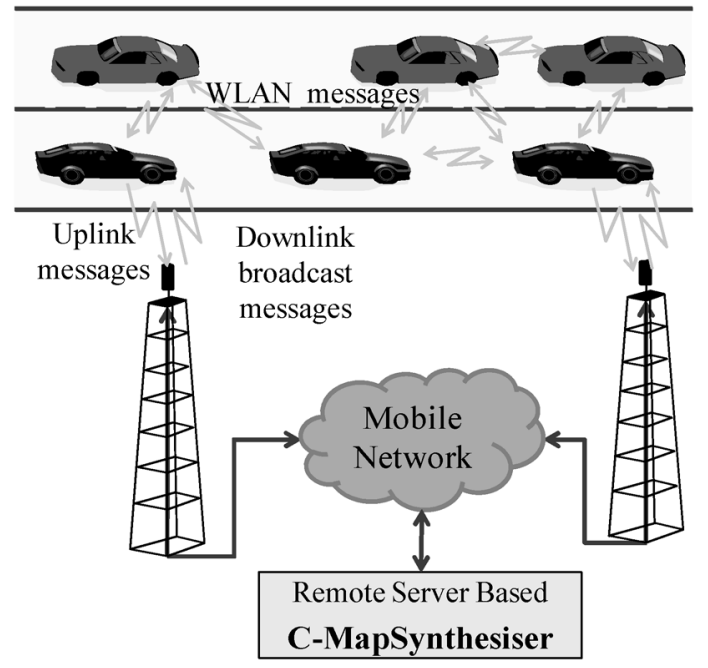

Fig. 5 Communication configurations

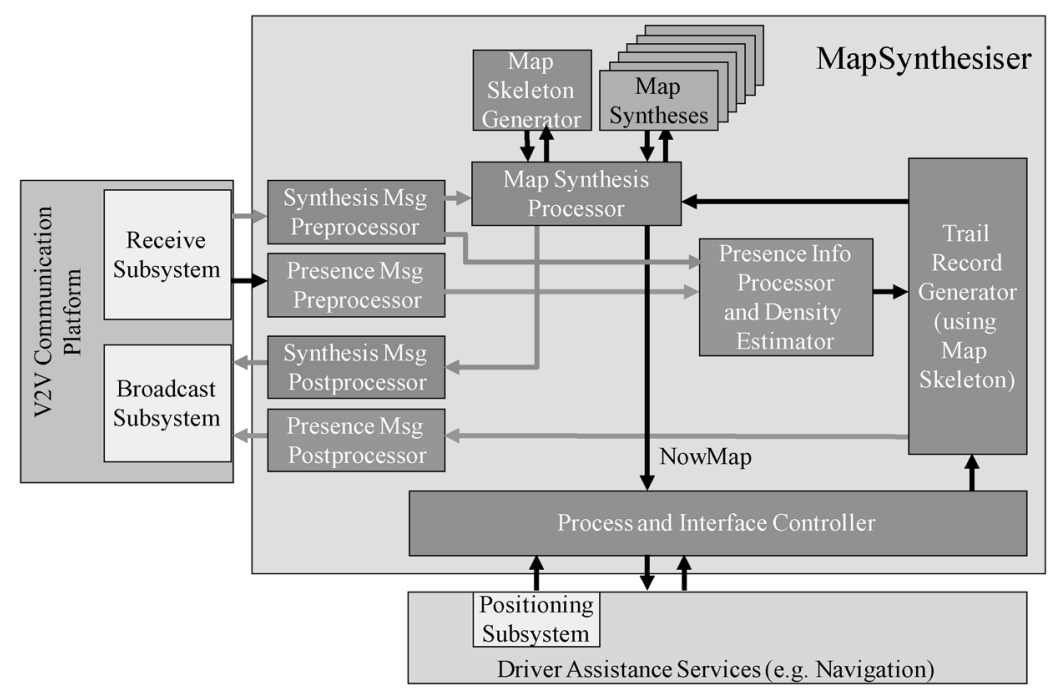

Fig. 4 MapSynthesiser block diagram 
frequency selection algorithm for broadcast. Furthermore, the choice of broadcast using the user datagram protocol makes a more elaborate communication protocol unnecessary. Since the payload for the UDP protocol is limited $(\sim 1.4 \mathrm{~kb})$ the map synthesis to be transmitted is dissected into a number of selfcontained chunks each fitting into the UDP messages payload.

The configurations discussed above are presented in Fig. 5. Even though a central system is not necessary for the functioning of GuideWeb, it can be enhanced with a remote server-based C-MapSynthesiser especially when widerange information coverage is desired. Similar remote serverbased systems have been analysed in [16]. C-MapSynthesiser is a server-based map synthesis processor. All received map syntheses are optimally allocated to C-MapSynthesiser areas and processed. The resulting map syntheses are broadcasted within the associated C-MapSynthesiser areas. Evidently, C-MapSynthesiser increases area coverage and GuideWeb performance.

\section{$5 \quad$ Processing map syntheses}

\subsection{Map synthesis attributes}

The map synthesis processor generates the map synthesis. From the received synthesis messages the relevant map synthesis information and additional information is extracted in a preprocessing stage. An example of an extracted map synthesis is shown in Fig. 6. On the right the map skeleton is presented, which summarises the retrieved knowledge about routes in the chosen environment. On the left the map synthesis is shown with the attributes for the route segments. For each segment of the map skeleton typically of length $100 \mathrm{~m}$ - the six values indicate average speed (AS), average density (AD) and traffic flow trend $(T)$ for each direction of the road are determined. Together with a weight indicating the relevance of the information, that is the older the information the less relevant it becomes, they form the route segment attribute.

The segment length of $100 \mathrm{~m}$ has been determined for reasons of compression of the geo-coordinates in synthesis messages. The compression algorithm employed allows exchanging information of $\sim 5000 \mathrm{~km}$ of roads in less than $100 \mathrm{~kb}$ messages. The MapSynthesiser adapts the covered area such that it contains $\sim 5000 \mathrm{~km}$. Thus, the diameter of the area varies according to the length of the road network within the area.
Further, the analysis of the data in MapSynthesiser uses statistical methods. Obviously, it is impossible to infer to a single contribution from a mean value. This ensures data privacy.

\subsection{Map synthesis history}

To generate the NowMap, the map synthesis processor collects and processes all received map syntheses for a defined time period, and keeps the history of the information of each route segment in memory as shown in Fig. 7.

The weights of the route segment attributes provide the time references to create this route segment history. They are simply numbers indicating the position, that is the 'age', of the corresponding data in the route segment history (see Fig. 7). The route segment history is used for computing the actual route segment attributes as well as for trend calculation if sufficient historical data are available. The history is stored in different levels, for example in level A the time difference of the attributes $A_{i}$ and $A_{i+1}$ is $1 \mathrm{~min}$, in level $B B_{i}$ and $B_{i+1}$ is $5 \mathrm{~min}$ and so on. The number of

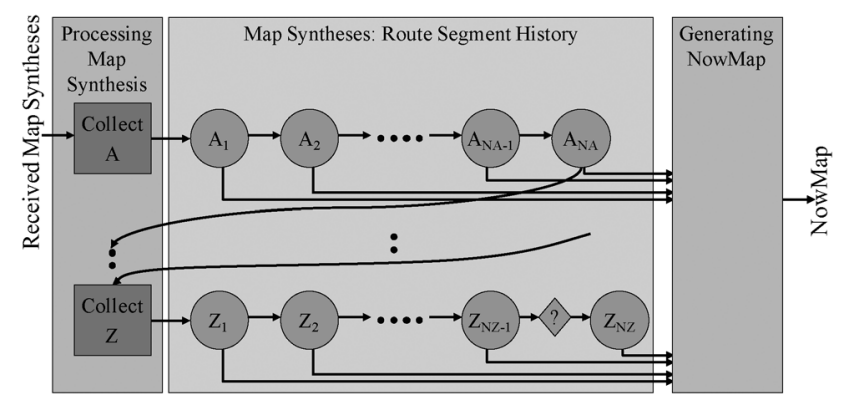

Fig. 7 Route segment history

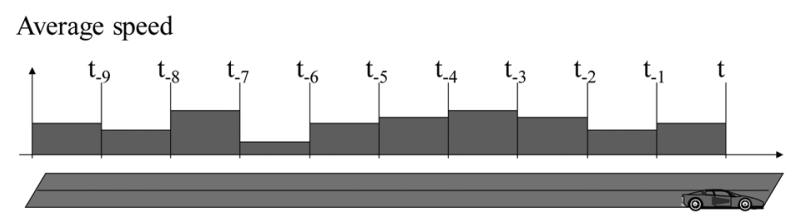

Fig. 8 Trail record contribution to map synthesis

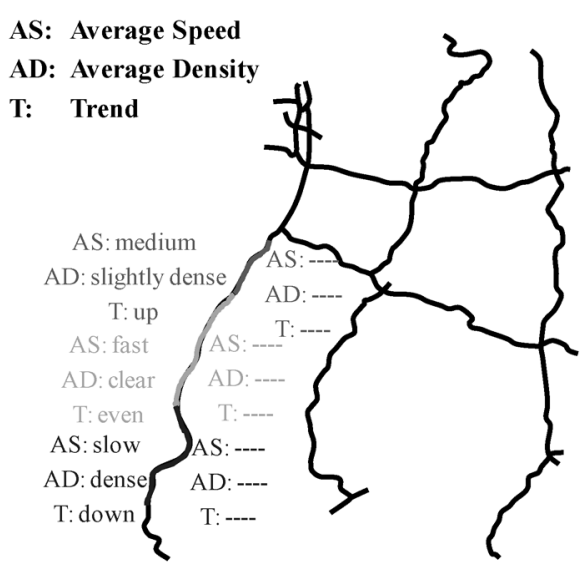

Map Synthesis

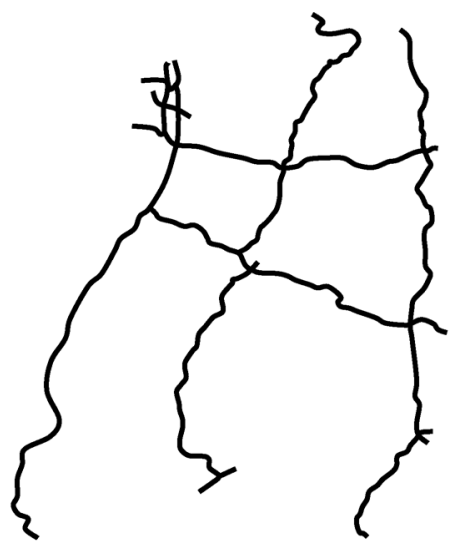

Map Skeleton

Fig. 6 Example of a map synthesis 
Table 1 Learn/forget cycle times against MapSynthesiser density

\begin{tabular}{lrrrrr}
\hline Percentage of vehicles with MapSynthesiser & $1.0 \%$ & $2.5 \%$ & $5.0 \%$ & $7.5 \%$ & $10.0 \%$ \\
\hline average time to learn, min & 30.40 & 23.72 & 18.62 & 15.94 & 14.11 \\
95\% confidence interval & \pm 2.00 & \pm 1.50 & \pm 0.87 & \pm 0.87 & \pm 0.43 \\
average time to forget, min & 39.57 & 28.66 & 16.90 & 14.05 & 12.41 \\
95\% confidence interval & \pm 3.57 & \pm 2.43 & \pm 0.88 & \pm 0.49 & \pm 0.29 \\
\hline
\end{tabular}

levels and the corresponding timing is a matter of map synthesis processor configuration.

Keeping the route segment history enables the map synthesis processor to perform plausibility checks to detect and eliminate unrealistic attribute values (e.g. introduced to the system by a malicious user) as well as to compute the attributes for NowMap taking into account historical attributes. Furthermore, the historical attribute data allow for a trend computation that indicates how traffic has evolved over time and what is to be expected.

\subsection{Generating trail record}

The most important ingredient to create a map synthesis is the trail record. A trail record is generated according to the following mechanism. When the process is started in a vehicle, the position is recorded with respect to the information received from the positioning subsystem, for example a global positioning system (GPS) receiver and the time. For a certain distance, for example $100 \mathrm{~m}$, called a trail segment, the instantaneous speeds of the vehicle are determined and their average is computed resulting in an average speed for this trail segment and forms the trail segment record together with traffic density information that is computed by counting the received presence and synthesis messages and inferred from this number and the speed indications. Then the process is started again.

The trail comprises the multitude of trail segments traversed and the trail record comprises the multitude of the corresponding trail segment records. The position indications of points of the trail segments, in particular the start point, provide the information of the form of the trail and the average speeds and densities on the trail segments provide suitable information to be advantageously processed for the map synthesis.

The vehicle under consideration generates a trail record (see Fig. 8), which it contributes to its present map synthesis. Initially that is without having received a synthesis message from another participating vehicle, a map synthesis consists of just the trail record. If speed is low, a time-out terminates and restarts this process after, for example $1 \mathrm{~min}$. In the case where the vehicle is not actively participating, for example a vehicle with switched-off engine, it may cease to contribute.

\section{Simulation}

GuideWeb has been simulated by an application-specific programme and some of the results are presented in this section. Most importantly, introducing route segment attribute historical data adds a level of complexity to the system that requires careful examination. On the one hand, GuideWeb needs to learn about a traffic situation and on the other hand, it must forget when this traffic situation changes.
This learning and forgetting is implemented in a LearnForget algorithm which has been simulated extensively. For ease of implementation the simulation has been based on a 'Manhattan' street model, that is the streets have a grid arrangement of 20 streets vertical and 20 streets horizontal. The distance between two neighbouring streets is $100 \mathrm{~m}$. This $100 \mathrm{~m}$ piece is called a segment. In total the grid has $20 \cdot 19=380$ segments and thus, a street length of $380 \cdot 100 \mathrm{~m}=38 \mathrm{~km}$. The vehicles drive randomly around in the grid and vehicles exchange map syntheses when they are within radio range thus simplifying the communication interface.

To introduce a traffic situation, a road block appears on one of the streets in one direction causing traffic congestion when cars move on this road in this direction. The simulation is performed in cycles, each cycle consisting of two phases.

- The first phase starts when the first 'car' learns about the congestion. It is completed when all vehicles know about the congestion, that is the NowMap of all cars indicate congestion on the corresponding route segments. Cars that know about the congestion will avoid it.

- The second phase starts with the removal of the roadblock and the traffic starts flowing again. It is completed when all vehicles have forgotten about the congestion, that is the NowMap of all cars indicate a flowing traffic on all route segments.

GuideWeb has been simulated according to the procedure outlined above with the following environmental parameter: number of vehicles $=1000$; radio reach $=100 \mathrm{~m}$ and the percentages of vehicles equipped with MapSynthesiser: 1, $2.5,5,7.5$ and $10 \%$. The number of cycles has been at least 100. The results are summarised in Table 1 and Fig. 9.

Obviously, as expected the average times to learn and to forget, respectively, decrease when the MapSynthesiser density increases.

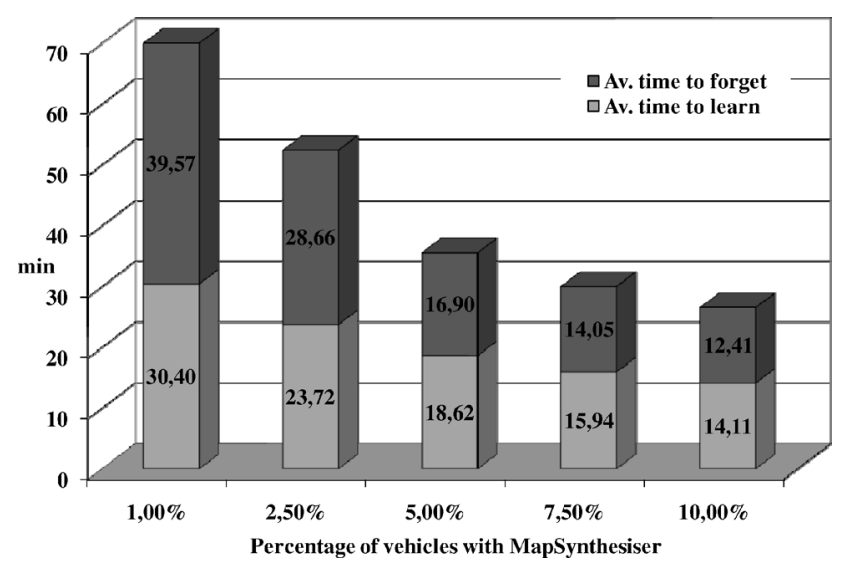

Fig. 9 Average learn/forget cycle times against MapSynthesiser density 


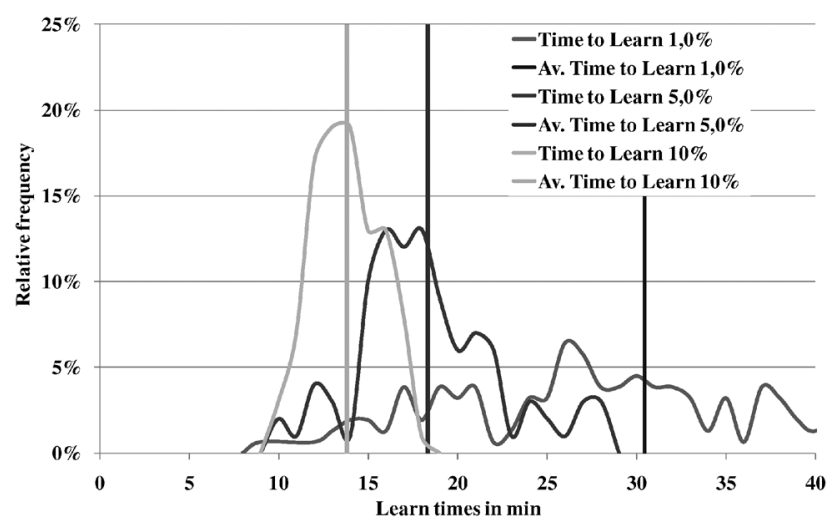

Fig. 10 Relative frequency of learn cycle times (MapSynthesiser density is parameter)

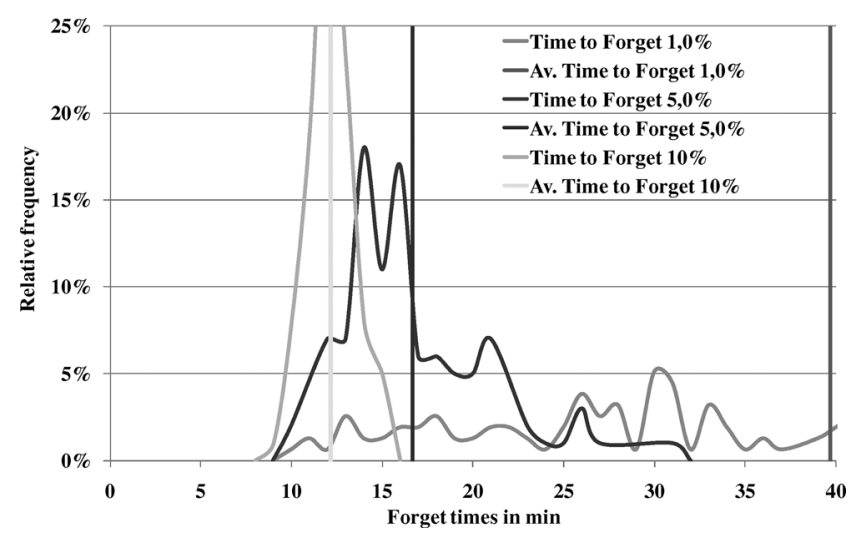

Fig. 11 Relative frequency of forget cycle times (MapSynthesiser density is parameter)

A very interesting insight from this simulation is that the system, that is the entirety of the vehicles and MapSynthesiser in the area, has a 'systemic memory'. The 'quality' of the systemic memory, that is the times for the system to learn and to forget about an incident, depends on the frequency of MapSynthesiser interaction. This is substantiated by the simulation results presented in Figs. 10 and 11. Relative frequency means in how many simulation cycles a time to learn and a time to forget, respectively, of $t+\Delta t$ appeared.

From Figs. 10 and 11 it is seen that

- average learn and forget times, respectively, indicated by vertical lines, become shorter as the density of MapSynthesiser increases as already stated above;

- the relative frequency function or histogram becomes sharper as density increases implying that learn and forget times become more predictable when more MapSynthesiser interactions are possible.

\section{Conclusion}

In this study the concept GuideWeb for vehicle navigation support based on $\mathrm{v} 2 \mathrm{v}$ communication has been presented. It has been shown that GuideWeb is a very suitable candidate for a commercially viable introduction of $\mathrm{v} 2 \mathrm{v}$ communication imposing a minimum requirement on networking. By using a broadcast communication concept, important difficulties in $\mathrm{v} 2 \mathrm{v}$ communication are overcome or circumvented, and because of its coordinate-based exchange format it is independent of map suppliers and easily integrated in any driver-assistance system. GuideWeb finds its application window beginning now until all the challenges of the $\mathrm{v} 2 \mathrm{v}$ networking capabilities are resolved.

Further, the insights derived from GuideWeb deployment and its behaviour allows learning about $\mathrm{v} 2 \mathrm{v}$ communication system performance. Keeping historical data of the route segment attributes adds a new level of complexity - the systemic memory. However, the advantages of historical data (detection of implausible attributes or malicious users, computing trends and so on) trade off favourably with the implications of the systemic memory as shown by simulation. Furthermore, by concept the penetration level required for GuideWeb to function is much lower than in other $v 2 v$ communication systems. The processing methodology allows that information about any specific vehicle can neither be extracted nor traced, that is that privacy is ensured.

\section{References}

1 Schnaufer, S., Füßler, H., Transier, M., Effelsberg, W.: 'Vehicular adhoc networks: single-hop broadcast is not enough'. Proc. 3rd Int. Workshop on Intelligent Transportation (WIT), Hamburg, Germany, March 2006, pp. 49-54

2 Torrent-Moreno, M.: 'Inter-vehicle communications: assessing information dissemination under safety constraints'. 4th Annual IEEE/ IFIP Conf. on Wireless on Demand Network Systems and Services (WONS), Obergurgl, Austria, January 2007

3 Baldessari, R., Festag, A., Abeille, J.: 'NEMO meets VANET: a deployability analysis of network mobility in vehicular communication'. Proc. 7th Int. Conf. on ITS Telecommunications (ITST 2007), Sophia Antipolis, France, June 2007, pp. 375-380

4 Bechler, M., Wolf, L., Franz, W.: 'Mobile internet access in FleetNet'. Proc. KiVS 2003, Leipzig, Germany, February 2003

5 Baldessari, R., Festag, A., Matos, A., Santos, J., Aguiar, R.: 'Flexible connectivity management in vehicular communication networks'. Proc. 3rd Int. Workshop on Intelligent Transportation (WIT), Hamburg, Germany, March 2006, pp. 211-216

6 Harsch, C., Festag, A., Papadimitratos, P.: 'Secure position-based routing for VANETs'. Proc. IEEE 66th Vehicular Technology Conf., (VTC Fall), Baltimore, MD, USA, September/October 2008

7 'Zukunft und Zukunftsfähigkeit der Informations- und Kommunikationstechnologien und Medien', Internationale DelphiStudie 2030, Münchner Kreis e.V., EICT GmbH, Deutsche Telekom AG, TNS Infratest GmbH, November 2009

8 EU-Project CVIS: http://www.cvisproject.org/

9 EU-Project FleetNet: http://www.fleetnet.de/

10 EU-Project Invent: http://www.invent-online.de/

11 German Research Project Network On Wheels: http://www.network-onwheels.de/

12 CAR 2 CAR Communication Consortium: http://www.car-2-car.org/

13 Wischhof, L., Ebner, A., Rohling, M., Halfmann, R.: 'SOTIS - a selforganizing traffic information system'. Proc. 57th IEEE Semiannual Vehicular Technology Conf. on VTC 2003-Spring, Jeju, South Korea, April 2003

14 Weis, J., Weis, P.: 'Device for exchanging data between moving vehicles'. European patent 1639566, 2008

15 ISO, Intelligent Transport Systems (ITS) - Location Referencing for Geographic Databases - Part 3: Dynamic Location References (Dynamic Profile), Standard 17572-3, 2009

16 Eliasson, C., Fiedler, M.: 'Dimensioning study for road user charging'. Proc. 15th ITS World Congress 2008, New York, USA, Novenber 2008 